\title{
Coração de Atleta em Desportistas Deficientes de Elite
}

\author{
Japy Angelini O liveira Fo, A ntonio Carlos Silva, Edgar Lira Fo, Bráulio Luna Fo, Silvia Helena Covre, \\ Flávio A ntônio A scânio Lauro, Marcelo Aria Dias Danucalov, Wagner Aparecido Leite, Sérgio Tuffik, \\ José Lázaro Andrade, Angelo A mato Vincenzo de Paola, Eulógio Martinez Foo
}

São Paulo, SP

Objetivo - Verificar a prevalência de sinais de "coração de atleta" em desportistas deficientes para-olímpicos.

Métodos - Avaliação clínica, eletrocardiográfica, ecocardiográfica, vetorcardiográfica, ergométrica em 75 atletas, 27,8 6 6,7 anos, 56 homens, várias modalidades; com deficiência física (47), visual (12) e paralisia cerebral (16).

Resultados - Sinais de coração de atleta ocorreram em $33 \%$ dos exames clínicos (sopros e estalidos), em $55 \%$ dos eletrocardiogramas (bradicardia, bloqueio incompleto de ramo direito, sobrecargas, alterações de onda T), em $15 \%$ dos vetorcardiograma (sobrecargas), em $5 \%$ dos ecocardiogramas (dimensões cavitárias acima do habitual). Os sinais ocorreram em $51 \%$ dos atletas, sendo que em $46 \%$ dos casos havia 2 ou mais sinais e, em 12\%, 4 ou mais sinais. O TE foi normal em 77\% dos atletas; não houve ST isquêmico. Em $23 \%$ dos casos houve bloqueio divisional direito.

Conclusão - Foram encontrados dois ou mais sinais de coração de atleta em $46 \%$ dos atletas deficientes paraolímpicos.

Palavras-chave: coração de atleta, atleta deficiente, eletrocardiograma, teste ergométrico, ecocardiograma

\section{Athlete's Heart in Elite Disabled Athletes}

Purpose - To assess the prevalence of the athlete's, heart syndrome in elite disabled Brazilians athletes.

Methods - Seventy-five athletes, age 27.8 \pm 6.7 years, 56 men, with various disabilities (47 physical, 12 visual and 16 cerebral paralysis) underwent clinical, electrocardiographic, vectorcardiographic, ergometric and echocardiographic evaluations.

Results - Athlete's heart signs occurred in $33 \%$ of the clinical evaluations, in $55 \%$ of the electrocardiograms, in $15 \%$ of the vectorcardiograms, and in $5 \%$ of the echocardiograms. At least one of these signs was presented in $51 \%$ of the athletes. There were 2 or more abnormalities in $46 \%$ of the athletes and 4 or more signs in 12\%. Exercise test was considered not ischemic in $77 \%$ of the subjects. There was right bundle branch block in $23 \%$ of the tests.

Conclusion - There were two or more athlete's heart syndrome signs in $46 \%$ of Brazilian disabled athletes.

Key-words: athlete's heart, disabled athletes, electrocardiogram, exercise test

Arq Bras Cardiol, volume 69 (nº 6$), 385-388,1997$

O coração de atleta é uma condição caracterizada por sinais clínicos, radiológicos, eletrocardiográficos e ecocardiográficos, descrita em atletas em atividade, traduzindo adaptações fisiológicas ao treinamento físico prolongado ${ }^{1-6}$. Descrito inicialmente por Henschen em $1899^{7}$, corresponde a adaptações cardiovasculares induzidas por uma interação de fatores centrais e periféricos, agindo em níveis estruturais, metabólicos, neurais e hormonais em substrato genéti-

Escola Paulista de Medicina - UNIFESP

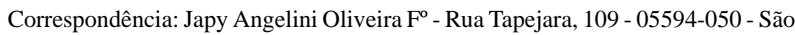
Paulo, SP

Recebido para publicação em 30/7/97

Aceito em 19/9/97 $\operatorname{co}^{8}$. Deficientes físicos apresentam peculiaridades com relação ao treinamento prolongado, relacionadas à perda de massa muscular, à incoordenação motora e à difícil adaptação às várias modalidades desportivas. Não se têm encontrado na literatura publicações específicas sobre coração de atleta entre deficientes. Estudamos uma série de casos consecutivos de desportistas deficientes para-olímpicos, em que foram pesquisadas a presença e as características do coração de atleta.

\section{Métodos}

Os autores estudaram 79 desportistas deficientes brasileiros para-olímpicos, de março a julho/96, sendo 49 defici- 
entes físicos, 12 deficientes visuais e 18 portadores de paralisia cerebral, com idade de 27,8 $\pm 6,7$ anos, 59 homens e 20 mulheres. Os atletas pertenciam a diferentes modalidades: futebol (18 atletas), natação (15), basquete (13), disco/dardo/peso (11), velocistas (6) e outras (16 atletas, incluindo: judô, 4 ; maratona, 2 ; ciclismo, 1 ; pentatlo, 1). Com relação à ocupação, 15 participavam apenas de atividades esportivas e 64 exerciam outras profissões.

Os desportistas foram submetidos a exame clínico, eletrocardiograma (ECG) convencional, ecocardiograma Doppler (ECO), reação sorológica para doença de Chagas (hemoaglutinação) e teste ergométrico (TE) em esteira ou cicloergômetro, adaptado o protocolo à modalidade esportiva e à deficiência física. Durante o TE, os registros foram realizados em CM5, sendo os atletas exercitados até a exaustão. Nos primeiros 63 casos foi realizado vetorcardiograma (VCG). Na avaliação do ECG foram utilizados os critérios de Romhilt-Estes para sobrecarga ventricular ${ }^{9}$. Todas as intervenções foram realizadas por observadores independentes. Em dois casos, o ECO foi repetido para confirmação diagnóstica, conhecendo o ecocardiografista o quadro clínico (atleta portador de insuficiência aórtica comprovada e atleta com suspeita de síndrome de Marfan afastada a posteriori); nestes dois casos, foi considerado apenas o resultado do primeiro exame.

Consideraram-se sinais de coração de atleta os seguintes achados: estalido sistólico, sopro sistólico, $3^{\mathrm{a}} \mathrm{e} 4^{\mathrm{a}}$ bulhas, bradicardia sinusal, arritmia sinusal, bloqueio incompleto do ramo direito (BIRD), sobrecarga ventricular padrão de repolarização precoce, padrão juvenil de onda T, diâmetros cavitários e espessuras ventriculares aumentadas ou no limite superior da normalidade no $\mathrm{ECO}^{4}$. Não se considerou a presença de coração de atleta os casos em que a única manifestação detectada foi a presença de estalido sistólico.

Após avaliação cardiológica excluíram-se da amostra quatro atletas com afecções cardiológicas comprovadas: hipertensão arterial (HA) e doença de Chagas forma indeterminada(1 caso), HA e insuficiência aórtica(1), hipertensão do jaleco branco - discutível (1) e doença de Chagas (1). Em todos esses casos, dados os graus discretos de repercussão clínica e a presença de coração, estruturalmente normal, não se constataram contra-indicações para a prática de esportes em níveis competitivos, sendo os atletas liberados à prática esportiva ${ }^{10-13}$.

\section{Resultados}

São relatados os resultados referentes aos 75 atletas sem afecções cardiológicas. No exame clínico, 50 (67\%) atletas apresentaram propedêutica normal. A propedêutica alterada ocorreu em 25, onde foram detectados: estalido sistólico mesocárdico(13 casos), estalido sistólico mitral, (4), sopro sistólico mitral (6), sopro sistólico aórtico (3), sopro sistólico mesocárdico (1), presença de $4^{\mathrm{a}}$ bulha (1). Em todos os casos, os sopros eram $+/++++++$ ou $++/+++++$ + e não guardavam correspondência com a presença de refluxos valvares mínimos no ECO.

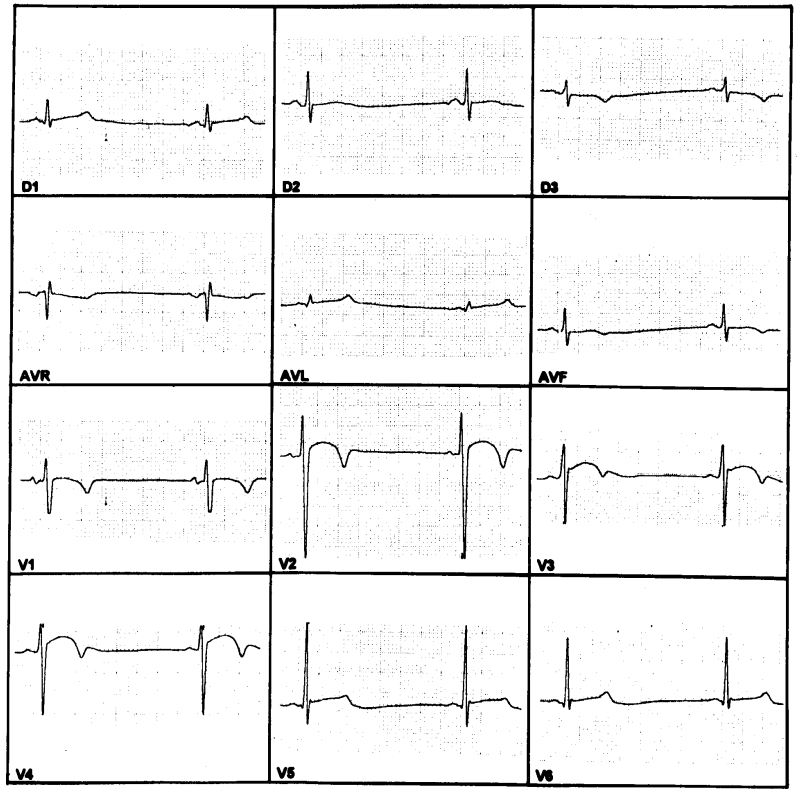

Fig. 1 - Atleta de 36 anos, sexo masculino, ciclista, frequiência cardíaca 38bpm, sobrecarga atrial esquerda, sobrecarga ventricular esquerda, padrão juvenil de onda T.

NoECG, 34 casos mostraram-se normais (47\%). Os traçados alterados apresentaram bradicardia sinusal (16 casos), bradiarritmias sinusal (10), arritmia sinusal (1), bloqueios divisionais (19 casos: anterior esquerdo, 1 atleta; posterior esquerdo, 1; superior direito, 9; posterior direito, 8), BIRD (1 caso), sobrecarga ventricular esquerda provável(1), padrão de repolarização precoce (29), onda T juvenil (2), PR curto (1) (fig. 1).

No VCG, detectaram-se alças normais em 27 (45\%) atletas, relatando-se 33 com alterações: bloqueios divisionais (27 casos: posterior esquerdo, 1; superior direito 11; posterior direito, 15), sobrecarga ventricular esquerda (4), sobrecarga ventricular direita (4), sobrecarga biventricular (1), perdas de forças septais (2).

No ECO, os diâmetros das cavidades e da aorta e a espessura do septo e parede livre de ventrículo esquerdo (VE) foram normais em todos os casos. Em quatro casos, os diâ-

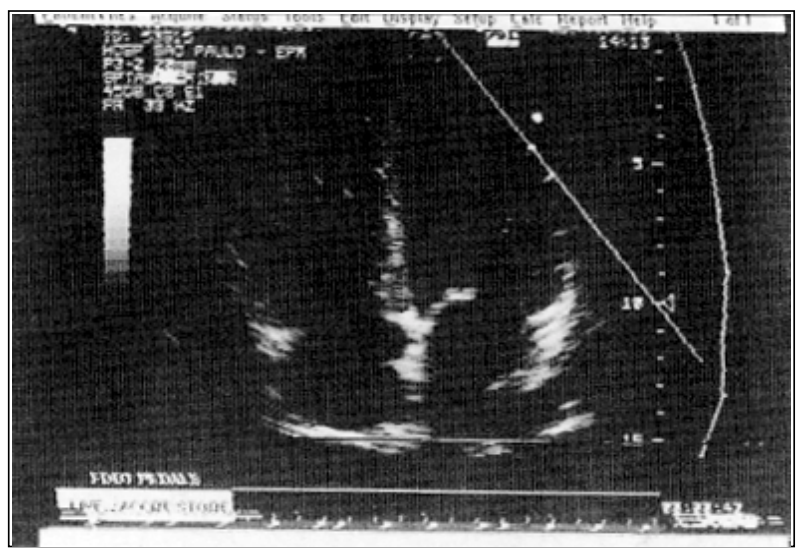

Fig. 2 - Atleta de 36 anos, sexo masculino, ciclista, diâmetro diastólico de ventrículo esquerdo $=52 \mathrm{~cm}$, diâmetro sistólico de ventrículo esquerdo $=34 \mathrm{~cm}$, septo $=11 \mathrm{~cm}$, ventrículo direito $=27 \mathrm{~cm}$, átrio esquerdo $=42 \mathrm{~cm}$. 


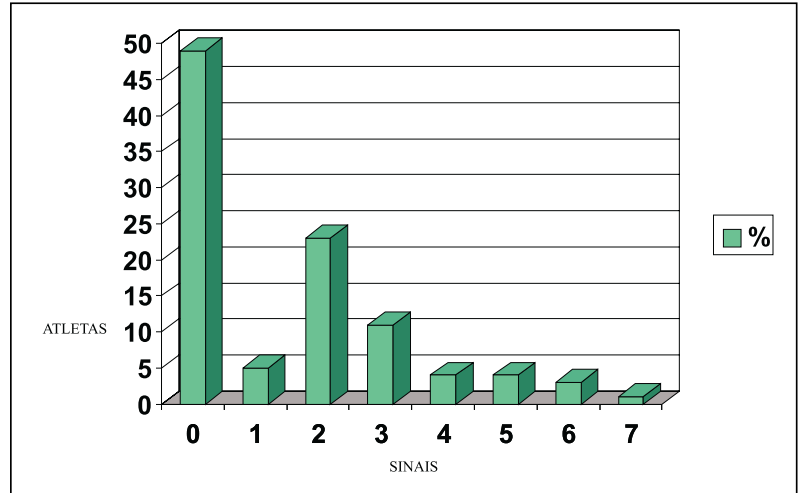

Fig. 3 - Gráfico indicativo do percentual de atletas com um ou mais sinais clássicos de coração de atleta.

metros situaram-se no limite superior da normalidade: (diâmetro diastólico de VE, 3 casos; diâmetro diastólico de VEe ventrículo direito (VD) e diâmetro de átrio esquerdo (AE), 1 caso). A espessura septal situou-se no limite superior da normalidade em dois e o diâmetro de aorta, em um. Refluxos valvares mínimos foram detectados em sete casos (mitral, 2; tricuspídeo, 5). A fração de ejeção e o percentual de encurtamento ventricular foram normais em todos os casos (fig. 2).

Durante o TE constataram-se respostas normais ao exercício em 58 (77\%) atletas. Em 15 casos descreveram-se as seguintes alterações: insuficiência cronotrópica (3), resposta hiper-reativa de pressão arterial (PA) (2), extra-sistolia ventricular isolada (5), extra-sistolia ventricular pareada (2), reversão de onda T (3). Em todos os casos as respostas de segmento ST foram normais.

Sinais de coração de atleta foram encontrados em 38 (51\%) casos: 1 sinal (4), 2 sinais (17), 3 sinais (8), 4 sinais (3), 5 sinais (3), 6 sinais (2), 7 sinais (1) (fig. 3 ).

\section{Discussão}

Inúmeras publicações na literatura têm descrito os sinais clínicos, eletrocardiográficos e ecocardiográficos da condição conhecida como coração de atleta. Na descrição original de Henschen ${ }^{7}$, a síndrome incluía bradicardia sinusal em repouso, sopro sistólico precoce, $3^{\mathrm{a}}$ e $4^{\mathrm{a}}$ bulhas, cardiomegalia avaliada pela radiografia de tórax e alterações do $\mathrm{ECG}^{4}$. Tendo em vista que os sinais avaliados, isoladamente, carecem de especificidade, no presente estudo, excluíram-se quatro atletas portadores de afecções cardiovasculares: $\mathrm{o} 1^{\circ}$, não apresentava sinais de coração de atleta, apenas níveis pressóricos elevados em repouso, resposta hipertensiva durante o TE e reações sorológicas positivas para doença Chagas, e controlada a HA, foi liberado para prática de competições ${ }^{11} ; \mathrm{o} 2^{\circ}$, apresentava HA e insuficiência aórtica, com duplo sopro aórtico, bloqueio divisional direito no ECG/VCG e resposta hipertensiva de PA no TE e o ECO mostrando função ventricular normal com dimensões situadas no limite superior da normalidade, sendo o atleta liberado após controle clínico ${ }^{11,13}, \mathrm{o} 3^{\circ}$, apresentava PA normal no exame clínico $(120 \times 75 \mathrm{mmHg})$ e níveis de PA elevados apenas no TE (controle 205x95 e pico
235x85mmHg). NoECG/VCG encontrou-se bloqueio divisional direito e no ECO as dimensões cardíacas foram próximas dos valores médios normais. Mantidos normais os níveis de PA, foi liberado para a prática esportiva ${ }^{11} ; 04^{\circ}$, apresentava sopro mitral, bradiarritmia sinusal, ECO normal, extra-sistolia ventricular pareada durante o TE e sorologia positiva para Chagas e, após avaliação clínica, foi liberado para atividades competitivas ${ }^{10,12}$. Assim, a exclusão dos quatro atletas, que, além dos sinais atribuíveis à afecção, poderiam apresentar coração de atleta, não afetou de forma significativa os resultados.

Os 75 atletas livres de afecções cardiovasculares apresentavam distintas deficiências em graus diversos, agrupadas em três condições: deficiências física e visual, e portadores de paralisia cerebral. Participavam de 14 diferentes modalidades predominantes, tornando a casuística bastante heterogênea e impedindo a formação de grupo controle adequado a desenho de estudo transversal.

Mostraram propedêutica cardiovascular normal 50 casos. Em sete casos a única alteração encontrada foi estalido sistólico; dada a falta de especificidade desse sinal clínico, estes atletas não foram considerados no grupo de desportistas com evidência(s) de coração de atleta. Em todos os casos, os sopros cardíacos não se acompanharam de refluxo valvar no ECO.

No ECG, 41 casos mostraram-se alterados. A prevalência de bradicardia sinusal (35\%) está de acordo com a literatura onde tem variado de 7,8 a $78,6 \%{ }^{14-17}$. Dada a falta de publicações, a incidência de bloqueios divisionais em atletas não está estabelecida; surpreendentemente a prevalência dos bloqueios divisionais direitos atingiu 23\%. No entanto, constatou-se apenas presença de um BIRD, estando publicado prevalência variável em atletas de $0,1 \mathrm{a} 14 \%{ }^{14}$. É provável que inúmeros casos classificados no presente estudo, como bloqueio divisional direito, tenham sido catalogados na literatura como BIRD. Desta forma os bloqueios divisionais direitos não foram incluídos como sinal de coração de atleta neste estudo. Em apenas um caso detectaramse evidências de sobrecarga ventricular esquerda. É possível que os critérios utilizados tenham interferido nos resultados ${ }^{9}$. No entanto fenômenos comuns aos deficientes físicos, como perda de massa muscular, incoordenação motora e, ainda, menor período de treinamento poderiam ter influenciado nesses resultados. Os dados referentes à repolarização precoce são similares aos descritos na literatura ${ }^{14}$.

No VCG realizado em 60 atletas sem evidências de afecções cardiovasculares, a incidência de alças anormais atingiu a 55\%, destacando-se a incidência de bloqueios divisionais direitos (43\%) e sobrecargas ventriculares $(15 \%)$. O achado de perda das forças septais esteve relacionado à presença de bloqueio divisional e não à sobrecarga ventricular esquerda.

NoECO, $5 \%$ dos atletas mostraram índices referentes a dimensões e espessuras cavitárias no limite superior da normalidade, aceitáveis como evidência de coração de atleta $^{4,18}$ ( 1 ciclista, 1 maratonista, 1 arremessador de peso, 1 judoca); neste subgrupo apenas um caso apresentou sinais 
vetorcardiográficos de sobrecarga ventricular. Todos os 75 atletas estavam em fase de treinamento ${ }^{19}$ e apresentaram, no ECO, coração estruturalmente normal. Os refluxos valvares mínimos não corresponderam à presença de sopros.

No TE, 77\% dos atletas mostraram resultados considerados compatíveis com a normalidade que compreenderam as respostas normais ao exercício, as respostas alteradas pela presença de extra-sistolia ventricular isolada e ou reversão da onda T. Jellinek e col ${ }^{20}$ relataram extra-sístoles ventriculares isoladas em 5\% da população normal. Batlouni ${ }^{21}$ relatou a normalização da onda T em futebolistas considerados sadios. Postula-se que as alterações de ST/T que ocorrem em atletas sem afecções cardiovasculares podem ser discriminadas pela regressão durante o exercício ${ }^{6,14,22}$. Em dois $(3 \%)$ casos a presença de extra-sistolia ventricular pareada ocorreu em atletas isentos de evidência de doença associada ou manifestações de overtraining ${ }^{23}$. Em população de indivíduos considerados sadios descreveu-se prevalência de $2 \%$ de extra-sístoles ventriculares pareadas ${ }^{20}$.

As manifestações típicas do coração de atleta têm sido descritas em sua maior expressão em atletas competitivos altamente treinados, pertencentes a modalidades que apresentam maiores exigências físicas: ciclismo de rua, esqui de longo curso, natação, remo, futebol ${ }^{6,8,22}$. Em nosso estudo, os atletas com maior número de sinais de coração de atleta pertenciam às seguintes modalidades: 7 sinais, 1 maratonista; 6 sinais, 1 ciclista, e 1 praticante de pentatlo; 5 sinais, 2 praticantes de atletismo e 1 judoca. Neste subgrupo, encontravam-se três dos quatro atletas com manifestações ecocardiográficas. É possível que sinais clínicos e eletrocardiográficos antecedam às manifestações detectadas no ECO. Neste estudo, $51 \%$ dos atletas apresentavam, ao menos, um sinal compatível com coração de atleta e $23 \%$ mostraram, pelo menos, três sinais referentes a esta condição.

Estudos futuros serão necessários para avaliar a prevalência do coração de atleta em desportistas deficientes de elite, separando-os por modalidade e deficiência; estudos que deverão ser obrigatoriamente multicêntricos envolvendo delegações para-olímpicas de diferentes países, classificando os atletas em subgrupos diversos, segundo modalidade esportiva e deficiência física. Além disso, haveria necessidade de grupo controle de deficientes físicos pareados por sexo, idade, deficiência física, origem e raça, que não estivessem participando de atividades desportivas regulares.

\section{Referências}

1. Morganroth J, Maran BJ - The athletes heart syndrome. A new perspective. Ann NY Acad Sci 1977; 301: 931-41.

2. Rost R - The athletes heart. Eur Heart J 1982; 3: 193-8.

3. Houston TP, Puffer JC, Rodney MW - The athletic heart syndrome. NEng J Med 1985; 4: 24-9.

4. Maron BJ - Structural features of the athlete heart as defined by echocardiography J Am Coll Cardiol 1986; 7: 190-203.

5. Crawford MH, O'Rourke RA - The athlete's heart. Adv Intern Med 1979; 24: 311-26.

6. Vivacqua R, Hespanha RF - Ergometria, Reabilitação e Cardiologia. Rio de Janeiro: Atheneu, 1994: 319-59.

7. Henschen SE - Skilauf und Skiwettlauf. Eine medizinishe Sportstudie. Mitt Med Klin (Upsalla) 1899; 2:15. Apud Hollmann W, Hettinger T - Medicina do Esporte. São Paulo: Manole, 1983.

8. Hollmann W, Hettinger T-Medicina do Esporte. São Paulo: Manole, 1983: 428-51

9. Romhilt DW, Estes Jr EH - A point-score system for the ECG diagnosis of left ventricular hypertrophy. Am Heart J 1968; 75: 752-8.

10. Maron BJ, Isner JM, McKenna WJ - Hypertrophic cardiomyopathy, myocarditis, and other myopericardial diseases and mitral valve prolapse. Med Sci Sports Ex 1994; 26(suppl): 261-7.

11. Kaplan NM, Deveraux RB, Miller Jr HS - Systemic hypertension. Med Sci Sports Ex 1994; 26(suppl): 268-70.

12. Zipes DP, Garson Jr A - Arrhythmias. Med Sci Sports Ex 1994; 26(suppl): 276-83.

13. Cheitlin Md, Douglas PS, Parmley WW - Acquired valvular heart disease. Med Sci Sports Ex 1994; 26(suppl): 254-60.
14. Zehender M, Meinertz T, Keul J, Hansjorg J - ECG variants and cardiac arrhytmias in athletes: clinical relevance and prognostic importance. Am Heart J 1990; 119: 1378-91.

15. Balady GJ, Cardigan JB, Ryan TT - Electrocardiogram of the athlete: an analysis of 289 professional football players. Am J Cardiol 1984; 53: 1339-43.

16. Batlouni M, Ghorayeb N, Armaganijan D, Meneghelo RS - Alterações eletrocardiográficas em atletas profissionais.II. Distúrbios do ritmo e da condução. Arq Bras Cardiol 1980; 35: 187-95.

17. Venerando A - Electrocardiography in sports medicine. J Sport Med Physiol Fitness 1979; 19: 107-28.

18. Pelliccia A, Maron BJ, Spataro A, Proschan MA, Spirito P - The upper limit of physiologic cardiac hypertrophy in highly trained elite athletes. N Engl J Med 1991; 324: 295-301.

19. Maron BJ, Pelliccia A, Spataro A, Granata M-Reduction in left ventricular wall thickness after deconditioning in highly trained olympic athletes. Br Heart J 1993; 69: 125-28.

20. Jelinek MV, Lown B - Exercise stress testing for exposure of cardiac arrhythmia. Prog Cardiovasc Dis 1974; 16: 497-522.

21. Batlouni M - Alterações eletrocardiográficas em atletas profissionais.III. Anomalias da repolarização ventricular. Arq Bras Cardiol 1980; 35: 387 95.

22. Duarte GM, Alfieri RG - Exercício e Coração. Rio de Janeiro: Cultura Médica, 1993: 458-77.

23. Lehmann M-Overtraining in endurance athletes: a brief review. Med Sci Sports Ex 1993; 25: 854-62. 